\title{
Effect of pirfenidone on cough in patients with idiopathic pulmonary fibrosis
}

\author{
To the Editor:
}

Idiopathic pulmonary fibrosis (IPF) is a progressive, fatal lung disease. In patients with IPF, cough is one of the most disabling symptoms and is an independent predictor of disease progression [1-3]. Cough in IPF is often non-responsive to antitussive therapy [4], and studies on cough are scarce and have unfortunately not yet resulted in effective treatments. Several observations suggest that pirfenidone, an anti-fibrotic drug, might decrease cough $[5,6]$. We aimed to measure objectively the effect of pirfenidone on cough in patients with IPF and substantial cough. In addition, we assessed the effect of pirfenidone on subjective cough and quality of life (QoL) measures.

This international, multicentre, prospective, observational study at four sites (The Netherlands, Italy, France and UK) recruited patients between 2013 and 2016. Treatment-naïve IPF patients aged 40-85 years with a forced vital capacity (FVC) $\geqslant 50 \%$ and corrected transfer factor of the lung for carbon monoxide $($ TLCOc) $\geqslant 30 \%$, in whom pirfenidone therapy was about to be initiated according to regular practice, who had daily IPF-related cough for $\geqslant 8$ weeks with a cough score of $\geqslant 40 \mathrm{~mm}$ on a $0-100 \mathrm{~mm}$ visual analogue scale (VAS), were eligible for the present study.

Patients underwent baseline 24-h cough recording with the Leicester Cough Monitor (LCM) prior to starting pirfenidone, and after 4 and 12 weeks of treatment. The LCM is a validated ambulatory cough monitoring system [7]. Recordings were centrally analysed with automated cough software as previously described [7]. On the days of cough recording, participants completed the Leicester Cough Questionnaire (LCQ, a cough-specific health status questionnaire), the VAS cough severity, VAS urge-to-cough, King's Brief Interstitial Lung Disease health status questionnaire (K-BILD), the Hospital Anxiety and Depression Scale (HADS) and the seven-item Generalised Anxiety Disorder screener (GAD-7) $[8,9]$.

Analyses were performed on the intention-to-treat population. Objective cough frequency data were log transformed. To assess the effect of pirfenidone on cough over time we used a linear mixed model, which accounts for missing data [10]. Sensitivity analyses were performed on the per protocol population with a linear mixed model and paired t-tests. SPSS Statistics version 21.0 and R version 3.2.2 software packages were used for statistical analysis. The study was approved by the ethics committees of all participating centres (clinical trials.gov, NCT02009293). All patients provided written informed consent.

Of the 46 patients screened for the study, 43 patients were included. Reasons for exclusion were low TLCOc and/or VAS scores. 38 patients completed 4 weeks of the study, and 31 completed 12 weeks. Reasons for dropout included: cessation of pirfenidone because of adverse events (8), logistic reasons (2), change of proton pomp inhibitor (1) and death (1). The age of the patients was $72 \pm 7$ (mean $\pm \mathrm{sD}$ ); most were men $(33,77 \%)$ and former smokers $(34,79 \%)$. Baseline cough counts, questionnaire scores and pulmonary function tests are shown in table 1 .

After 12 weeks of pirfenidone treatment, objective 24 -h cough decreased by $34 \%$ (95\% CI $-48 \%$ to $-15 \%$ ) (table 1). An improvement in 24-h cough was observed in 20 out of 27 patients (74\%). Sensitivity analysis showed similar results (data available on request). Subjective cough measures showed consistent improvements (table 1). No significant changes in disease-specific QoL and anxiety were found. Even at the earlier time point of 4 weeks, a smaller, but significant effect on cough counts was observed, with a $14 \%$ reduction in $24-\mathrm{h}$ cough frequency $(95 \% \mathrm{CI}-22 \%$ to $-6 \% ; \mathrm{p}=0.002)$. At this time point, improvements in cough were observed in 24 out of 35 patients $(69 \%)$.

@ERSpublications

In patients with IPF, pirfenidone reduces objective 24-h cough counts and improves subjective measures of cough http://ow.ly/iiGu30f7Pea

Cite this article as: van Manen MJG, Birring SS, Vancheri C, et al. Effect of pirfenidone on cough in patients with idiopathic pulmonary fibrosis. Eur Respir J 2017; 50: 1701157 [https://doi.org/10.1183/ 13993003.01157-2017]. 
TABLE 1 Effect of 12 weeks of pirfenidone treatment on objective and subjective cough and health status measures, analysed with a linear mixed model

\begin{tabular}{lcccc} 
& Baseline & At 12 weeks & Change $^{\#}$ (95\% CI) & p-value \\
\hline Subjects n & 43 & 31 & & \\
24-h cough & $520(91$ to 3394$)$ & $392(75$ to 1746$)$ & $-34 \%(-48$ to $-15 \%)$ & 0.002 \\
Coughs per hour & $23(4$ to 141$)$ & $17(3$ to 73$)$ & $-35 \%(-49$ to $-17 \%)$ & $<0.001$ \\
$\quad$ Daytime & $28(5$ to 171$)$ & $20(4$ to 121$)$ & $-33 \%(-47$ to $-14 \%)$ & 0.003 \\
$\quad$ Night-time & $7.2(0.7$ to 101$)$ & $3.3(0$ to 54$)$ & $-34 \%(-54$ to $-5 \%)$ & 0.029 \\
LCQ & $12 \pm 4$ & $15 \pm 4$ & $2.0(1.0 \text { to } 3.0)^{\text {\% }}$ & $<0.001$ \\
VAS cough & $67 \pm 15$ & $47 \pm 27$ & $-19(-28$ to -10$)$ & $<0.0001$ \\
VAS urge-to-cough & $68 \pm 16$ & $49 \pm 25$ & $-18(-26$ to -10$)$ & $<0.0001$ \\
K-BILD total & $50 \pm 22$ & $55 \pm 23$ & $3.4(-2.3$ to 9.1$)$ & 0.245 \\
HADS anxiety & $8.5 \pm 4$ & $8.5 \pm 4$ & $0.7(-0.6$ to 1.9$)$ & 0.291 \\
HADS depression & $4.7 \pm 3$ & $6.0 \pm 3$ & $1.6(0.5$ to 2.6$)$ & 0.004 \\
GAD-7 & $5.8 \pm 6$ & $5.9 \pm 6$ & $0.7(-0.9$ to 2.3$)$ & 0.396 \\
FVC \% pred & $78 \pm 15$ & $79 \pm 17$ & & \\
TLCOc \% pred & $51 \pm 13$ & $51 \pm 16$ & &
\end{tabular}

Data are presented as median (range) or mean \pm SD, unless otherwise stated. For the Leicester Cough Questionnaire (LCQ) and the King's Brief Interstitial Lung Disease health status questionnaire (K-BILD), a higher score indicated better disease-specific quality of life/cough-related quality of life. For all other variables, a higher score indicated worse cough frequency/lurge-to-Jcough/anxiety/depression. VAS: visual analogue scale; HADS: Hospital Anxiety and Depression Scale; GAD-7: Generalised Anxiety Disorder seven-item scale; FVC: forced vital capacity; \% pred: \% predicted; TLcoc: corrected transfer factor of the lung for carbon monoxide. ${ }^{\#}$ : analysed using a linear mixed model, change in \%, points or $\mathrm{mm}$; ${ }^{\text {ๆ: }}$ minimal clinical important difference for chronic cough is 1.3 .

In this observational study, pirfenidone treatment reduced objective 24-h cough counts and subjective cough severity, and improved cough-related QoL in patients with IPF. The magnitude of these changes was clinically meaningful to patients [9].

This study is the first to show a significant improvement in cough by a pharmacologic treatment, using both objective and subjective measures in IPF patients. Pirfenidone's anti-fibrotic effect was thought to be enigmatic, but has recently been linked to the inhibition of GLI transcription factors $[5,11]$. Whether this inhibition relates to the antitussive effect of pirfenidone is unclear. In IPF, increased cough reflex sensitivity is thought to be a key mechanism that causes cough [4]. Preliminary studies in guinea pigs show that pirfenidone decreases cough reflex sensitivity [6]; however, its effect on cough reflex sensitivity in patients with IPF is yet unknown.

Recently, mechanical tissue stretch was shown to activate TGF- $\beta 1$ and contribute to the development of pulmonary fibrosis [12]. LesLIE et al. [13] proposed that recurrent stretch injury, caused by pressure changes during breathing, promotes fibrosis. Cough might be an additional source of mechanical stress, and could theoretically contribute to a pro-fibrotic feedback loop. This hypothesis, if correct, might explain why cough independently predicts disease progression [3]. More research is needed to investigate the potential role of cough as a driver of disease progression in IPF, as this might have therapeutic implications.

Only patients with a VAS cough score $\geqslant 40 \mathrm{~mm}$ were included in our trial, likely accounting for the higher cough frequencies we found, compared to those reported by KEY et al. [2] (median cough/h: 23 versus 9.4). The pattern of higher daytime cough frequency was similar to that of other studies in IPF and chronic cough [2]. Inhibition of cough by cortical pathways and higher thresholds of peripheral nerves at night, in addition to less exposure to stimuli that induce cough, might account for this pattern.

Although the treatment of gastroesophageal reflux disease (GERD) reportedly does not affect cough, the current study required patients with GERD to be on a proton pump inhibitor and remain on a stable dose [14]. We found no significant changes in K-BILD scores, which is likely due to the fact that the K-BILD assesses general QoL issues related to IPF and not specific symptoms. Depression scores, but not anxiety, increased after 12 weeks of treatment; however, the score remained below the cut-off of eight indicating signs of depression. Therefore, the clinical relevance of this finding remains unclear.

Studies evaluating antitussive treatment in IPF are scarce. HorTon et al. [15] found that thalidomide improved cough-specific QoL in IPF patients, although no objective cough measurements were assessed. 
Furthermore, thalidomide is known to have a toxic side-effect profile. In six IPF patients in an uncontrolled study, high dose oral corticosteroids improved cough, but not cough-related QoL [4]. Moreover, immunosuppression has been associated with deleterious effects in IPF. To date, no studies have assessed the effect of the anti-fibrotic drug nintedanib on cough. Our study results might imply that pirfenidone is preferable for treatment of IPF patients with cough; however, it would be valuable to compare the effects of nintedanib and pirfenidone on cough.

Our study has some limitations. As the study was not controlled, the impact of a placebo is not known. When this study was designed, pirfenidone had been just recently made available. This created high expectations, and therefore patients, physicians and the ethics committee considered it unethical at the time to deny patients pirfenidone treatment for 12 weeks, a decision that might be viewed differently nowadays. The only placebo-controlled pharmacologic study in IPF that used objective cough as an outcome, showed no placebo effect [16]. We therefore believe that the outcomes of our study are not significantly influenced by a placebo effect, which is further supported by the already significant, though smaller effect on cough after 4 weeks of treatment. Furthermore, we included IPF patients with a VAS score of $\geqslant 40 \mathrm{~mm}$. Therefore, we do not know the effect of pirfenidone on IPF patients with mild cough. Lastly, our study had a short follow-up period. The long-term effect of pirfenidone on cough in patients with IPF is therefore unknown; however, our results suggest that the effect of treatment on IPF cough could be explored in 12-week trials.

In conclusion, in patients with IPF, pirfenidone treatment significantly reduces objective 24-h cough counts by $34 \%$, and improves subjective measures of cough. These results are clinically meaningful to patients. More research is warranted into the mechanisms and management of cough in IPF and other fibrotic diseases.

Mirjam J.G. van Manen ${ }^{1}$, Surinder S. Birring ${ }^{2}$, Carlo Vancheri ${ }^{3}$, Virginia Vindigni ${ }^{3}$, Elisabetta Renzoni ${ }^{4}$, AnneMarie Russell ${ }^{4,5}$, Monique Wapenaar ${ }^{1}$, Vincent Cottin $\odot^{6}$ and Marlies S. Wijsenbeek ${ }^{1}$

${ }^{1}$ Dept of Respiratory Medicine, Erasmus University Medical Center, Rotterdam, The Netherlands. ${ }^{2}$ Division of Asthma, Allergy and Lung Biology, King's College London, London, UK. ${ }^{3}$ Dept of Clinical and Experimental Medicine, Section of Respiratory Disease, University of Catania, Catania, Italy. ${ }^{4}$ Interstitial Lung Disease Unit, Royal Brompton Hospital, London, UK. ${ }^{5}$ National Heart and Lung Institute, Imperial College London, London, UK. ${ }^{6}$ Dept of Respiratory Medicine, Louis Pradel Hospital, Hospices Civils de Lyon, Claude Bernard Lyon 1 University, Lyon, France.

Correspondence: Marlies S. Wijsenbeek, Dept of Respiratory Medicine, Erasmus University Medical Center, Rotterdam, The Netherlands. E-mail: m.wijsenbeek-lourens@erasmusmc.nl

Received: Jan 132017 | Accepted after revision: July 172017

Support statement: The study was funded by an unrestricted investigator-initiated grant from InterMune/Roche. The funding source had no input in the design, data collection and analysis and writing of the manuscript, or in the decision to submit the manuscript for publication. Funding information for this article has been deposited with the Crossref Funder Registry.

Conflict of interest: Disclosures can be found alongside this article at erj.ersjournals.com

Acknowledgements: The authors thank Marion Durand (Louis Pradel Hospital, Hospices Civils de Lyon, Lyon, France), Marjolein Gerrits (Erasmus University Medical Center, Rotterdam, The Netherlands) and Claire Wood (King's College London, London, UK) for clinical research assistance, and Daan Nieboer (Erasmus University Medical Center) for statistical support. Author contributions: S.S. Birring, C. Vancheri, V. Cottin and M.S. Wijsenbeek designed the study. M.J.G. van Manen, C. Vancheri, V. Vindigni, E. Renzoni, A. Russell, V. Cottin and M.S. Wijsenbeek were involved in data collection. M.J.G. van Manen, S.S. Birring, M. Wapenaar and M.S. Wijsenbeek performed data analyses. M.J.G. van Manen and M.S. Wijsenbeek drafted the manuscript. All authors interpreted the data and worked collaboratively to review and prepare the final article.

\section{References}

1 Center for Drug Evaluation and Research (CDER), U.S. Food and Drug Administration (FDA). The voice of the patient; a series of reports from the U.S. Food and Drug Administration's (FDA's) Patient-Focused Drug Development Initiative. Idiopathic Pulmonary Fibrosis. March 2015. www.fda.gov/downloads/ForIndustry/ UserFees/PrescriptionDrugUserFee/UCM440829.pdf Date last updated: March 2015. Date last accessed: January 2017.

2 Key AL, Holt K, Hamilton A, et al. Objective cough frequency in idiopathic pulmonary fibrosis. Cough 2010; 6: 4.

3 Ryerson CJ, Abbritti M, Ley B, et al. Cough predicts prognosis in idiopathic pulmonary fibrosis. Respirology 2011; 16: $969-975$

4 Hope-Gill BDM, Hilldrup S, Davies C, et al. A study of the cough reflex in idiopathic pulmonary fibrosis. Am J Respir Crit Care Med 2003; 168: 995-1002.

5 Azuma A, Taguchi Y, Ogura T, et al. Exploratory analysis of a phase III trial of pirfenidone identifies a subpopulation of patients with idiopathic pulmonary fibrosis as benefiting from treatment. Respir Res 2011; 12: 143. 
6 Okazaki A, Ohkura N, Fujimura M, et al. Effects of pirfenidone on increased cough reflex sensitivity in guinea pigs. Pulm Pharmacol Ther 2013; 26: 603-608.

7 Birring SS, Fleming T, Matos S, et al. The Leicester Cough Monitor: preliminary validation of an automated cough detection system in chronic cough. Eur Respir J 2008; 31: 1013-1018.

8 Patel AS, Siegert RJ, Brignall K, et al. The development and validation of the King's Brief Interstitial Lung Disease (K-BILD) health status questionnaire. Thorax 2012; 67: 804-810.

9 Raj AA, Pavord DI, Birring SS. Clinical cough IV: what is the minimal important difference for the Leicester Cough Questionnaire? Handb Exp Pharmacol 2009; 187: 311-320.

10 Chakraborty $\mathrm{H}, \mathrm{Gu} \mathrm{H}$. A mixed model approach for intent-to-treat analysis in longitudinal clinical trials with missing values. RTI Press 2009; MR-0009-0903: 1-10.

11 Didiasova M, Singh R, Wilhelm J, et al. Pirfenidone exerts antifibrotic effects through inhibition of GLI transcription factors. FASEB J 2017; 31: 1916-1928.

12 Froese AR, Shimbori C, Bellaye PS, et al. Stretch-induced activation of transforming growth factor-betal in pulmonary fibrosis. Am J Respir Crit Care Med 2016; 194: 84-96.

13 Leslie KO. Idiopathic pulmonary fibrosis may be a disease of recurrent, tractional injury to the periphery of the aging lung: a unifying hypothesis regarding etiology and pathogenesis. Arch Pathol Lab Med 2012; 136: 591-600.

14 Kilduff CE, Counter MJ, Thomas GA, et al. Effect of acid suppression therapy on gastroesophageal reflux and cough in idiopathic pulmonary fibrosis: an intervention study. Cough 2014; 10: 4.

15 Horton MR, Santopietro V, Mathew L, et al. Thalidomide for the treatment of cough in idiopathic pulmonary fibrosis: a randomized trial. Ann Intern Med 2012; 157: 398-406.

16 Birring SS, Wijsenbeek MS, Agrawal S, et al. A novel formulation of inhaled sodium cromoglicate (PA101) in idiopathic pulmonary fibrosis and chronic cough: a randomised, double-blind, proof-of-concept, phase 2 trial. Lancet Respir Med 2017; 5: 806-815. 